Section 4.

\title{
TIME AND STANDARDS
}




\title{
Time and Standards - An Overview
}

\author{
Bernard Guinot \\ Observatoire de Paris \\ 61, avenue de l'Observatoire, 75014 - Paris, France
}

\begin{abstract}
After recalling current performances of time and frequency standards and their use to establish time scales, their limitations in astronomical observations and studies are considered. For expressing the value of almost all quantities, the accuracy of the frequency standards is sufficient. However, an improved stability could find applications in observation techniques and the departure of realized time scales with respect to their theoretical counterparts is not negligible for some studies. The paper recalls the basic rule of quantity calculus and advocates its use in spite of its inadequacy to express difference of readings of time scales. In general, adherence to the metrological practice and the use of the SI may facilitate our work and its understanding by the non-astronomers.
\end{abstract}

\section{Introduction}

The progress of the measurement of frequency and time is directly or indirectly the source of better measurements in astrometry referred to better realizations of space and time reference systems, requiring accurate modelling. Have the frequency stability and accuracy reached a level of quality which exceeds needs in astrometry? The paper is an attempt to answer this question. It also examines some metrological aspects in astronomical work.

\section{Accuracy and stability of frequency standards}

When dealing with frequency standards, one has to distinguish two basic characteristics: frequency accuracy and frequency stability.

In a broad sense, frequency accuracy of a frequency standard is its ability to produce a frequency in known relationship with the frequency of a selected transition for a non-perturbed atom or molecule, without external calibration. In the context of dynamical astronomy and astrometry, a more restrictive definition is the ability of a standard to measure proper time and proper frequency in conformity with the definition of the SI second, without external calibration.

For about two decades before 1998, the inaccuracy in frequency of the best standards (primary standards) remained at the level of about $2 \times 10^{-14}$, in relative value. Then, progress was resumed with fountains of cold cesium atoms, first developed at the Laboratoire Primaire du Temps et des Fréquences (LPTF), then in several laboratories, with inaccuracy in the range 1 to $2 \times 10^{-15}$. Currently, these devices are subject to further improvement and function episodically; one 
has still to rely on cesium fountains functioning more frequently, or even permanently, with inaccuracy of about $1 \times 10^{-14}$. All these standards are on the ground. Better results are expected in space, where standards using cold cesium atoms might have an inaccuracy of $1 \times 10^{-16}$ in the project ACES on the International Space Station. The use of other devices might reduce the inaccuracy to $10^{-16}$ to $10^{-17}$ even on the ground. The possibility of such an inaccuracy for a standard based on the locked rotation of the atomic ion-plasma has been mentioned by the National Institute of Standards and Technology (NIST).

In the observations for the dynamics of the solar system, accuracy in frequency is always required since it ensures the accuracy of numerical values of quantities, either observed or derived from the observations, in SI units, and a convenient realization of the theoretical time scales. For the observations, there is currently no need of inaccuracy smaller than $10^{-12}$ to $10^{-13}$. This can be easily obtained using calibration of a sufficiently stable standard by an external source (such as information received from the GPS and from the Bureau International des Poids et Mesures, BIPM) or by use of industrially made cesium standards. A more demanding application of the frequency accuracy is the realization of time scales, as will be shown later.

Frequency stability is the ability of a frequency standard to keep its mean frequency over a stated interval of time $\Delta \tau$, without precise reference to the second. The instability is a function of $\Delta \tau$ usually defined as the Allan standard deviation $\sigma_{y}(\Delta \tau)$, either under its original form (Rutman, 1978), or a modified form (Sullivan et al., 1990). (In the time community, $\Delta \tau$ is designated as $\tau$.)

The need for stable frequencies appears in observation techniques, in real time. Therefore only the frequency stability of some standards available for applications will be considered. As shown by Figure 1, for short time, up to about 1 second, quartz oscillators are the best. The averaging time of about 1 hour to one day is the domain of hydrogen masers, with an optimum of about $\sigma_{y}(\Delta \tau)=5 \times 10^{-16}$. For the long term, over a few days, industrially made cesium standards are convenient. For these instruments, Figure 1 shows the instability stated by the maker, but better stability may be expected in good laboratory environments. The long-term stability is especially useful to keep the synchronization of clocks and for realizing time scales. The improvement of stability, especially for short and medium term, might have an impact on the quality of astronomical observations, particularly in VLBI. Unfortunately, the progress in stability is slow. Over the very long term, years, decades, keeping the accuracy is the only way to maintain the stability, the instability and the inaccuracy taking about the same values.

\section{Time scales, International Atomic Time}

It is straightforward to extend the frequency characterization to a scale of proper time by considering the frequency standard, real or fictitious (such as an average over the data of a set of local standards), which produces it. Thus, the notions of frequency accuracy and stability can be applied. In addition the phase noise has to be characterized. As the realization of a time scale involves an integration over frequency, a strongly correlated noise with reinforcement at low Fourier frequency is generated. Even if purely random, this noise is very apt to resemble 


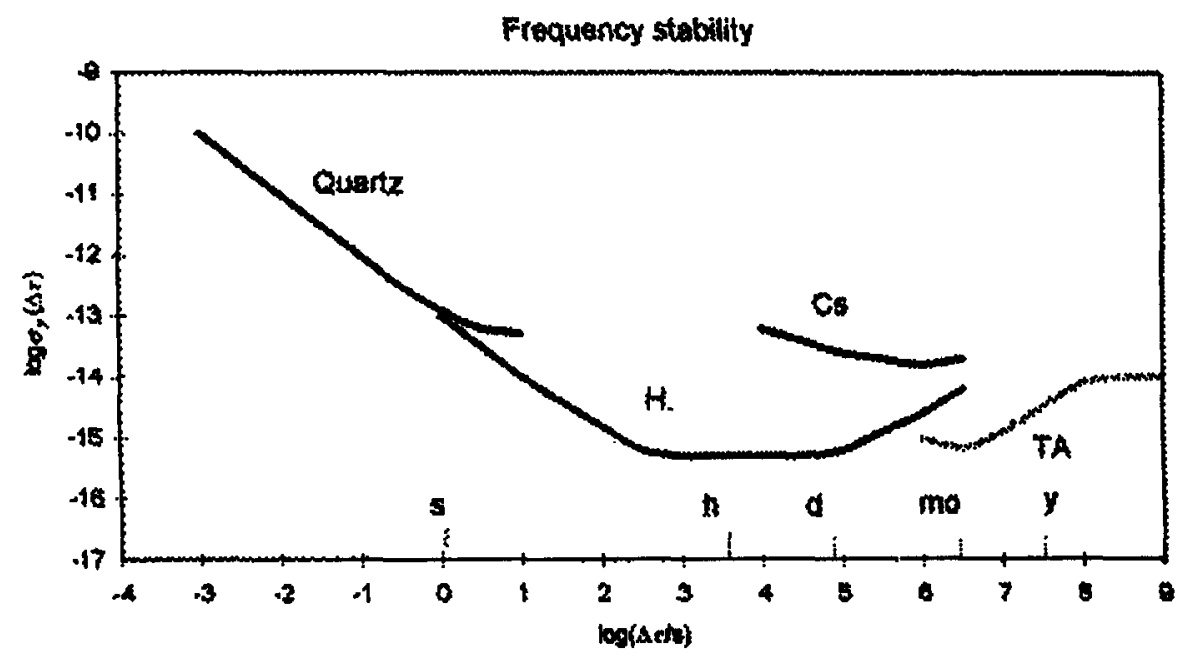

Figure 1. Instability of frequency standards available for applications and of TAI (assuming, for TAI, that its present characteristics are kept indefinitely).

a drift and long-period oscillations. The Allan standard deviation in phase $\sigma_{x}(\Delta \tau)$ is proportional to $\Delta \tau \times \sigma_{y}(\Delta \tau)$.

For a realized coordinate time, the noise characteristics are those of the estimated difference between the ideal scale and its realization. They cover the sum of: (a) the noise of the proper time of the clock from which the coordinate time is derived, (b) the noise of the transformation from proper time to coordinate time, (c), in some cases, the noise of time transfers. It is desirable that the inaccuracy brought by (b) and (c) be negligible (say an order of magnitude smaller than that of the clock). The frequency stability also should not be degraded in the whole range of averaging time, particularly in the long term.

International Atomic Time, TAI, is a realization of the ideal Terrestrial Time, TT, (with an historical time offset of $32.184 \mathrm{~s}$ ) defined by the IAU Resolution A4 (1991). The scale unit is somewhat loosely defined by the condition that it be equal to the SI second on the rotating geoid. At the current level of accuracy, this condition is still sufficient to establish TAI from the proper time of some 200 clocks and from about 10 primary frequency standards, all situated on the ground. However, with the rapid progress in accuracy, it becomes urgent that the theoretical and practical relation between proper time of terrestrial and space clocks with TAI be ensured with uncertainty in frequency at the $10^{-17}$ to $10^{-18}$ level $\left(1 \times 10^{-18}\right.$ is the gravitational shift for $1 \mathrm{~cm}$ elevation at the surface of the Earth). This problem, which may involve an abandonment of the reference to the geoid, is being considered by the BIPM/IAU Joint Committee on Relativity.

The frequency inaccuracy of TAI reported by the BIPM is currently $1 \times$ $10^{-14}$. The frequency instability is shown by Figure 1, assuming that present properties are kept indefinitely. In phase, the departure of TAI from its ideal 
counterpart may not be negligible. To get an idea of its amount, one can consider the difference between TAI $(+32.184 \mathrm{~s})$ and another representation of TT, TT(BIPM99), established in 1999 by the BIPM by reprocessing the same data as for TAI, using information obtained in retrospect as explained in Guinot (1988). Prior to 1977.0, TAI was a free running time scale, its scale unit being not constrained to represent accurately the second on the geoid. At 1977.0, the relative departure of the scale unit of TAI from the best realizations on the second (referred to the geoid) was about $1.0 \times 10^{-12}$. At 1977 January 1 , the scale unit of TAI was corrected by exactly this amount, and a steering process based on the data of primary standards was implemented. Nevertheless the difference between TT(BIPM99) and TAI $+32.184 \mathrm{~s}$ increases up to $25 \mu \mathrm{s}$ (Figure 2). The difference is larger than the uncertainty of UT1, for example, and much larger than the uncertainty of the time of arrival of pulses of millisecond pulsars. This is mainly due to the omission of a correction for black body radiation in cesium standards, which was controversial and not applied by all laboratories operating primary standards until 1996. One can also notice an annual variation with peak-to-peak amplitude of almost $1 \mu$ s in 1977 , which progressively vanished. The cause of that irregularity was probably a sensitivity to humidity of industrially made cesium clocks.

These errors on TAI cannot be corrected in retrospect because TAI is, for practical reasons, definitive as soon as it is made available by monthly Circular $\mathrm{T}$ of the BIPM with a delay ranging from 3 to 8 weeks. The improvement of the frequency accuracy is not superfluous in astronomy.

\section{Coordinated Universal Time}

Although it is a matter of seconds of time rather than microarcseconds, I would like to make two remarks on the Coordinated Universal Time UTC, in preparation for future discussions.

First, one should note that the 22 leap seconds introduced from 1972 to 2000 have all been positive and that the general trend is that they remain positive with an increasing frequency of occurrence. In 1970, the CCIR has recommended a rule (which later became Recommendation IUT-R TF.460-4) for dating events without ambiguity in the vicinity of a leap second. However this rule is valid only when using dates in hours, minutes and seconds. In all decimal systems of dating an ambiguity remains in case of a positive leap second. Consider an example in the vicinity of the positive leap second which occurred on 1999 January 1. Dates 1998 December 31, 23m 59m 60.5s UTC and 1999 January 1,

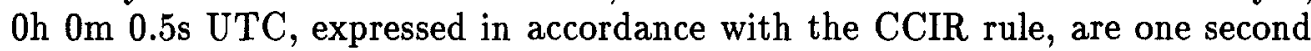
apart. However, they both receive the date $\mathrm{MJD}$ (UTC) $=51179.00000579$.

The second remark is that the decade fluctuations of UT1 have been surprisingly smooth since 1955, when atomic time became available. Figure 3 has been established simply by taking the values of UT1-TAI on the 1st of January. A prediction of UT1-TAI over two years would never have been in error by more than 1 second. 


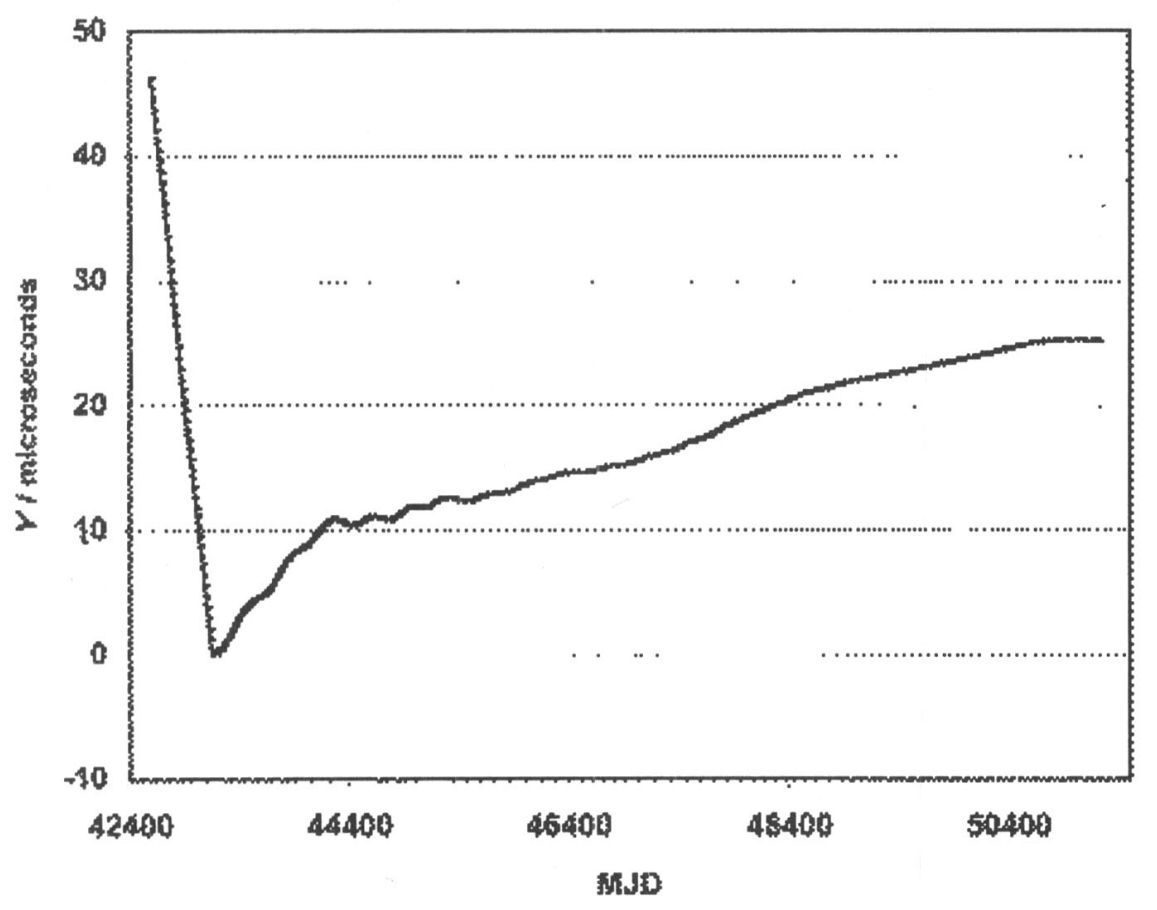

Figure 2. Difference between two realizations of TT: TAI +32.184 $s$ and TT(BIPM99) (by courtesy of the BIPM).

\section{Time, units and constants}

The accuracy of the realization of the second invites us to define other base units by linking them to the second, using physical laws and constants. This raises practical and theoretical questions.

Practically, this type of definition can be adopted only if it leads to an improvement in the realization of the units. Take the example of the definition of the meter by fixing the value of the velocity of light $c$. This definition was possible because mesurements of frequency of infra-red and visible radiations were achieved, hence providing accurate wavelengths used for the realization of the meter by interferometry. In contrast, defining the kilogram through a defining value of $G$ would lead to accurate masses of the celestial bodies, but without precise relation to laboratory masses. Experiments aiming at linking the kilogram to the second through electromagnetic and electrostatic forces are being considered. A better definition of electrical units may be based on quantum effects leading to frequency measurements (the Josephson and Hall effects), but the need to maintain the link between the mechanical and electrical units (the mechanical watt and electrical watt have to be equal) has, up to now, prevented defining electrical units through these effects. There is however a possibility 


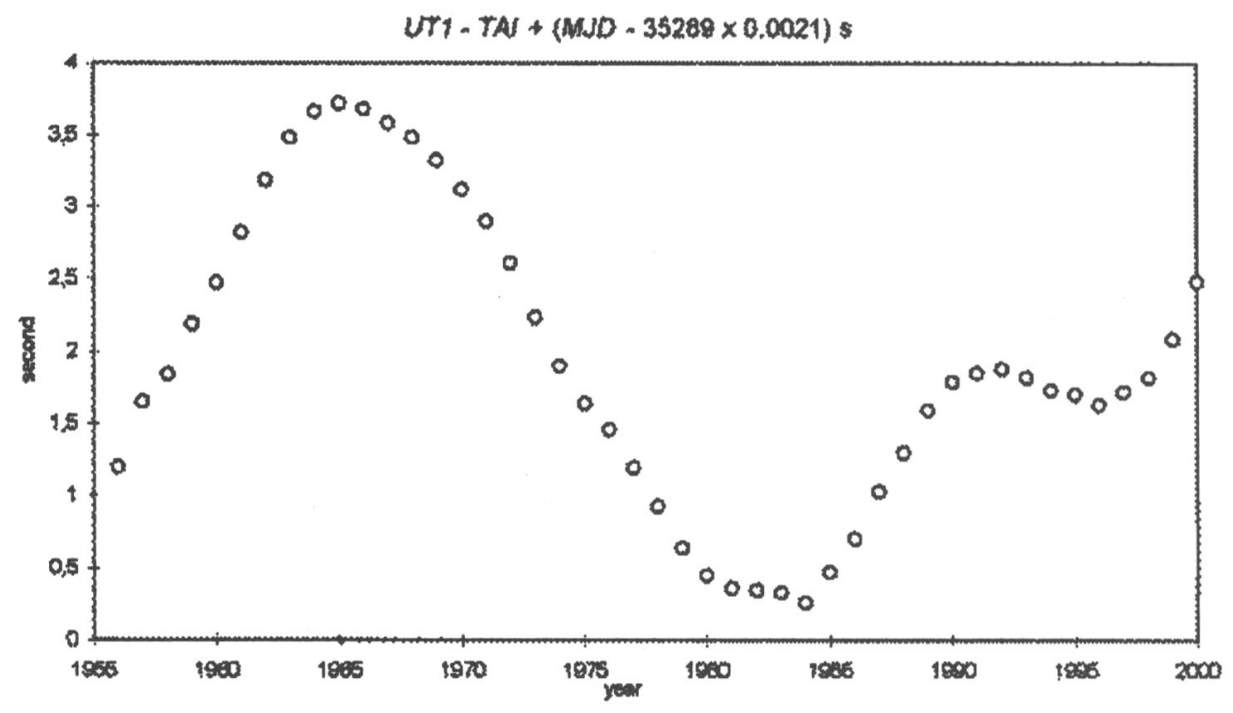

Figure 3. Values of UT1-TAI on the 1st of January (corrected by a linear function of time).

that future definitions of all base units, with the exception of the mole and the kelvin, and be based on the definition of the second.

Physical units should not depend on the choice of a reference system. They are defined as proper quantities. In metrological developments, it is assumed that local physics is the same everywhere and at anytime - the Einstein's Equivalence Principle (EEP). However, it has been sometimes argued that general relativity is a theory for macroscopic physics and that the EEP may not be applicable to atomic constants which intervene in the definition of the second and could be used in future definition of other SI units. Although no inconsistency has been found, these possibilities have to be kept in mind.

\section{Time and astronomical quantities}

\subsection{SI and astronomical units}

For the ephemerides of the solar system, two systems of units are employed: the SI and the astronomical units. Both use the same definition of the second. Basically, they differ by the choice of the unit of length. In the SI the value of $c$ is a defining constant of that unit, while $G M_{S u n}$ is left to experimental determination; in astronomical units, $G M_{S u n}$ is the defining constant for length and $c$ is measured. For the theory, both systems are equivalent and in both, the realization of the unit of time does not contribute significant uncertainties in the values of constants, parameters and various quantities. The risk of difficulties emanates rather from the choice of coordinate systems. It may be lessened by adhering to the usual metrological rules for expressing quantities, which will be examined in 6.2. 
The astronomical unit of length has played an essential role. Is it still needed as a unit independent from the meter? Would not it be possible to abandon it for ephemeris work and to provide it (as well as the parsec) by a fixed relation with the meter for other purposes?

\subsection{Expression of quantities}

The use of Dynamical Barycentric Time TDB instead of Coordinate Barycentric Time TCB has sometimes led to the introduction of new units for time and length, the so-called TDB-units, in replacement of SI units (associated with TCB). For example $G M_{S u n}$ is seen as a single quantity which receives different values in TDB-units and in SI-units. This point of view is in contradiction with the rule of quantity calculus which requires that all quantities having the same dimension be expressed in a single unit system, the SI (de Boer, 1995). According to that rule, the use of TDB and TCB leads to different quantities, with different notations, expressed in SI units. In the preceding example, one may distinguish $\left(G M_{S u n}\right)_{T D B}$ associated with TDB and $\left(G M_{S u n}\right)_{T C B}$ associated with TCB, both expressed in $\mathrm{m}^{3} \mathrm{~s}^{-2}$.

The classical rule in metrology avoids confusion and its use should be recommended. Nevertheless, we have to recognize that it leads to difficulties of logic with the readings of coordinates and scales, especially, but not exclusively, with relativistic coordinates. That will be illustrated by coordinate time. The date of an event in coordinate time is expressed in seconds (or multiples), which is correct in quantity calculus. However one cannot avoid giving a special name to the interval between two consecutive second markers of a stated coordinate time. For example, the BIPM publishes the duration of the TAI scale interval in SI seconds on the rotating geoid. Often the expression scale unit is taken as a synonym of scale interval. These scale units or intervals should be wellidentified quantities which can be measured in SI proper seconds under specified circumstances (specified event).

Another difficulty is the expression of difference of readings of two time scales $\mathrm{A}$ and $\mathrm{B}$ at the same event, usually under the form:

$$
A-B=x \text { seconds at event } \mathrm{E} \text {. }
$$

There is no duration involved in the left part of the expression, but the second is defined as the duration of... There had been long discussions on this topic which ended on account of lassitude of the protagonists, without a clear conclusion. Some pragmatism is needed here: everyone understands the above notation, let us consider it as conventional and let us forget about its metrological and philosophical interpretation.

\subsection{Quantities and metric}

Most of the quantities appearing in celestial mechanics and ephemerides are coordinate dependent. They should be defined in conformity with the metric adopted by the IAU. The increasing theoretical complexity makes this requirement even more important. One might assume that the coordinate system to which such quantities pertain is implicit. However, ambiguities may arise, especially with the space-time coordinate systems associated with TDB and TT. It would be much better to specify explicitly the coordinate system which is used. 
This is especially important for space geodesy, because in this discipline we do measure lengths and the ambiguities may be of the same size as the effects we intend to measure, for example in satellite altimetry. Let us recall that it is not possible to reconcile simultaneously the scale units for coordinate length and coordinate time with the proper meter and second on the rotating geoid by simply using a single scale factor in the metric (such as by the use of TT). Here, the use of the IAU metric, recognized by the IUGG, without scaling factors (i.e. with TCG) should be strongly recommended.

\section{Conclusion}

The metrology of time fulfills almost all the needs of dynamical astronomy and astrometry. Nevertheless a few questions and limitations remain. The most fundamental aspect is the ability of atomic frequency standards to provide the proper time of dynamical theories - a permanent problem until the answer no is provided either by the usual ephemeris work or by dedicated experiments. We have seen that a better frequency stability may improve the measurements and that a better accuracy would regularize the realized time scales, which is important for pulsar studies, based either on their rotation or on their orbital motion.

There is also a domain where it might be possible to bring improvements: the metrological aspect of our work. I would like to express the personal view that the rigor of theoretical developments and their increasing complexity calls for a better observance of metrological rules. It might be advisable also to use more extensively the SI, to reduce the number of units (especially for angles), to avoid specialized and non-decimal units. Metrology provides a common language, which could facilitate understanding between astronomers, geodesists, space research engineers, and physicists.

\section{References}

de Boer J., 1995, Metrologia, 32, 405-429.

Guinot B., 1988, Astron. Astrophys., 192, 370-373.

Rutman J., 1978, Proc. IEEE, 66, 1048-1075.

Sullivan D.B., Allan D.W., Howe D.A. and Walls F.L., (eds), 1990, Nist Technical Note 1337. 